\title{
Acquired von Willebrand syndrome: focused for hematologists
}

\author{
Massimo Franchini ${ }^{1}$ and Pier Mannuccio Mannucci ${ }^{2}$ \\ ${ }^{1}$ Department of Transfusion Medicine and Hematology, Carlo Poma Hospital, Mantua and \\ ${ }^{2}$ Fondazione IRCCS Ca' Granda Ospedale Maggiore Policlinico, Angelo Bianchi Bonomi \\ Hemophilia and Thrombosis Center, Milan, Italy
}

Haematologica 2020

Volume 105(8):2032-2037

\section{Correspondence:}

PIER MANNUCCIO MANNUCCI

piermannuccio.mannucci@policlinico.mi.it

Received: April 14, 2020.

Accepted: May 27, 2020.

Pre-published: June 18, 2020.

doi:10.3324/haematol.2020.255117

Check the online version for the most updated information on this article, online supplements, and information on authorship \& disclosures: www. haematologica.org/content/105/8/2032

(C)2020 Ferrata Storti Foundation

Material published in Haematologica is covered by copyright. All rights are reserved to the Ferrata Storti Foundation. Use of published material is allowed under the following terms and conditions:

https://creativecommons.org/licenses/by-nc/4.0/legalcode. Copies of published material are allowed for personal or internal use. Sharing published material for non-commercial purposes is subject to the following conditions:

https://creativecommons. org//icenses/by-nc/4.0/legalcode, sect. 3. Reproducing and sharing published material for commercial purposes is not allowed without permission in writing from the publisher.

\section{ABSTRACT}

T he acquired von Willebrand syndrome (AvWS) is a rare bleeding disorder with laboratory findings similar to those of inherited von Willebrand disease. However, unlike the inherited disease, AvWS occurs in persons with no personal and family history of bleeding and is often associated with a variety of underlying diseases, most frequently lymphoproliferative, myeloproliferative and cardiovascular disorders. After the presentation of a typical case, in this narrative review we discuss the more recent data on the pathophysiology, clinical, laboratory and therapeutic aspects of this acquired bleeding syndrome. We chose to focus particularly on those aspects of greater interest for the hematologist.

\section{Introduction}

Acquired von Willebrand syndrome (AvWS) is a rare but probably underestimated bleeding disorder characterized by laboratory findings and clinical presentations similar to those of inherited von Willebrand disease (vWD) ${ }^{1.8}$ Differing from vWD, a bleeding disorder due to quantitative or qualitative genetic defects of von Willebrand factor (vWF),, 10 AvWS usually occurs more frequently in adults with no personal or family history for a bleeding diathesis. Although it was first recognized more than 50 years ago (it was described in 1968 in a patient with systemic lupus erythematosus), AvWS has gained renewed interest in the last few years due to its association with relatively frequent cardiovascular disorders, including congenital heart defects, aortic stenosis, and the use of left ventricular assist devices. ${ }^{11-15}$ In addition to these, many other underlying diseases are associated with AvWS, ranging from solid and hematologic cancers to autoimmune diseases. ${ }^{16-18}$ Various mechanisms are implied in the pathophysiology of AvWS, the majority of them leading to the increased degradation or clearance of circulating vWF. This article reviews current knowledge on the mechanisms, diagnostic, clinical and therapeutic aspects of AvWS, focusing particularly on those cases associated with hematologic disorders. AvWS associated with cardiovascular diseases is not discussed here because it requires particular diagnostic and treatments strategies which were extensively and recently analyzed. ${ }^{11-15,19,20} \mathrm{~A}$ brief description of an individual case provides an example which allows us to introduce the main characteristics and management of the syndrome.

\section{Clinical case}

A 70-year old man presented to the emergency room of the main Mantua city hospital in north east Italy with spontaneous gingival bleeding. Apart from mild fatigue and headache, the patient felt well, with no bruising or other hemorrhagic symptoms. His medical history was positive for hypertension under satisfactory drug control but negative for a bleeding diathesis, and he had undergone an inguinal herniotomy 20 years earlier with no hemorrhagic complications. On physical examination, there was mild cutaneous and conjunctival pallor, blood oozing from the gums, and lymphadenomegaly at superficial stations (maximum diameter, $2 \mathrm{~cm}$ ). Blood tests revealed normocytic anemia (hemoglobin $9 \mathrm{~g} / \mathrm{dL}$ ), with normal white cell and platelet counts. With a normal prothrombin time, the activated partial thromboplastin time (APTT) was mildly prolonged (ratio, 1.29; normal range, $0.82-1.18$ ), but its full correction with a normal plasma mixing test excluded 
a coagulation inhibitor. Screening for lupus anticocoagulant was also negative. Factor VIII coagulant activity (FVIII:C) was $40 \%$ (normal range, 50-150\%), von Willebrand factor antigen (vWF:Ag) was 18\% (normal range. 50-120\%), ristocetin co-factor activity (vWF:RCo) was $29 \%$ (normal range, 50-150\%), and the collagen binding activity (vWF:CB) was 37\% (normal range, 50-150\%). Following the observation of slightly elevated serum proteins ( $8.8 \mathrm{~g} / \mathrm{dL}$; normal range, 6.5-8.0 g/dL), electrophoresis showed increased concentrations in the beta $(\beta)(2.58$ $\mathrm{g} / \mathrm{dL}$; normal range, 0.6-0.9 g/dL) and gamma $(\gamma)(2.36$; normal range, 0.8-1.4 g/dL) regions, with a double spike at a concentration of $1.67 \mathrm{~g} / \mathrm{dL}$ (Figure 1). Immunofixation confirmed a double monoclonal component, IgM kappa (к). Immunoglobulin assays showed serum IgG levels of $5.39 \mathrm{~g} / \mathrm{L}$ (normal range, 7-16 g/L), IgA $0.11 \mathrm{~g} / \mathrm{L}$ (normal range, 0.7-4 g/L), but very high IgM at $63.7 \mathrm{~g} / \mathrm{L}$ (normal range, $0.4-2.3 \mathrm{~g} / \mathrm{L}$ ). Bone marrow biopsy detected increased cellularity (90\%) that accounted for at least $40 \%$ of interstitial cellular aggregates of lymphoid, lymphoplasmacytoid and plasma cells, that at immunohistochemical analysis were positive for CD20, IgM and $\kappa$ light chains but negative for CD5, CD23, D1 cyclin and lambda $(\lambda)$ light chains. Megakaryocytic and myelo-erythroid lineages were represented but depressed. An abdominal ultrasound showed no hepatosplenomegaly nor lymphadenopathy. On the basis of these findings a diagnosis of AvWS associated with Waldenstrom macroglobulinemia was made. The patient underwent a test with desmopressin (DDAVP) given subcutaneously at a dose of 0.3 $\mu \mathrm{g} / \mathrm{kg}$ in an attempt to increase vWF and FVIII plasma levels, but no increase was observed at 1, 2 and 4 hours post injection. Due to the very high serum levels of the IgM monoclonal component, the patient underwent four plasma apheretic procedures (each with the removal of 1.5 plasma volume), resulting in a significant reduction in the monoclonal component, and normalization of vWF/FVIII parameters (vWF:Ag 86\%, vWF:RCo 77\%, vWF:CB 69\%, and FVIII:C $84 \%$ ). The patient was treated with six monthly cycles of bendamustine-rituximab, with a good partial response (IgM $6 \mathrm{~g} / \mathrm{L}$, normal hemoglobin and

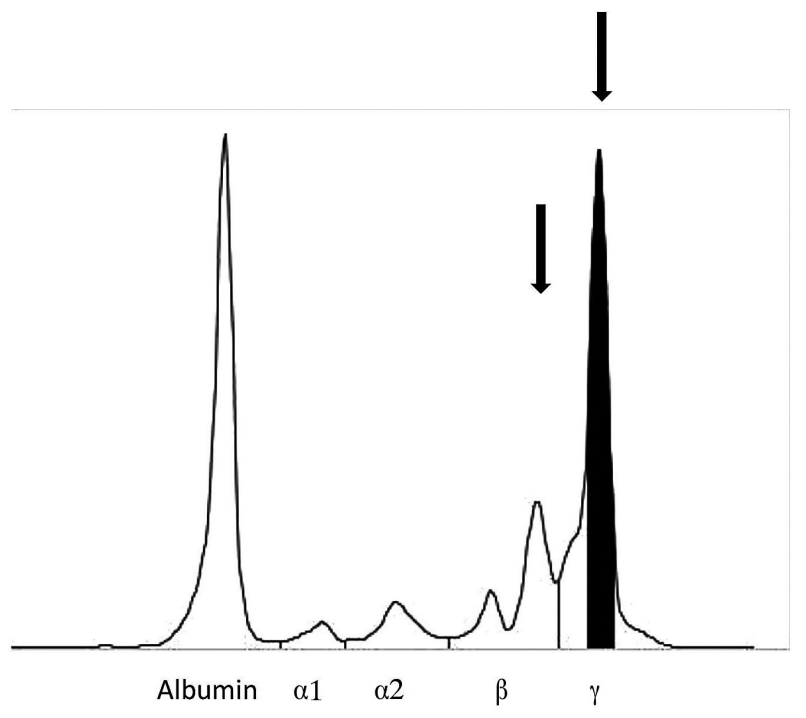

Figure 1. Example clinical case of acquired von Willebrand syndrome. Patient's serum protein electrophoresis. The arrows indicate a double spike within the beta $(\beta)$ and gamma $(\gamma)$ regions, respectively. hemostasis test values, complete disappearance of the enlarged lymph nodes). This positive response was maintained at a 3-year follow up, and a recent bone marrow biopsy showed a normal trilineage hematopoiesis with less than $10 \%$ lymphoplasmacytoid cells.

\section{Pathophysiology, clinical presentation, diagnosis}

\section{Pathophysiology}

In the past, AvWS was considered a very rare hemorrhagic disease. The more recent discovery of its association with relatively frequent cardiovascular disorders suggests that its prevalence is higher than previously thought. ${ }^{19,20}$ Unlike acquired hemophilia (another rare acquired bleeding disorder caused by autoantibodies that neutralize FVIII coagulant activity), ${ }^{21-23}$ the complex pathophysiology of AvWS involves various and different mechanisms. ${ }^{16-18}$ Most cases are due to an increased plasma clearance of vWF caused by such mechanisms as antibodies, cell adsorption, shear stress or increased proteolysis. At variance with acquired hemophilia, AvWS almost always occurs in association with an underlying disorder; ${ }^{16-18}$ besides cardiovascular disorders, those more frequently associated are lymphoproliferative disorders [multiple myeloma, chronic lymphocytic leukemia, monoclonal gammopathy of undetermined significance (MGUS), Waldenstrom macroglobulinemia], and, less frequently, other hematologic malignancies (myeloproliferative neoplasms including essential thrombocythemia, polycythemia vera, primary myelofibrosis, and chronic myeloid leukemia), solid malignancies, and autoimmune disorders (Table 1). ${ }^{16-18,24,25}$ In a registry of the International Society of Thrombosis and Haemostasis (ISTH) that collected data from 211 AvWS cases, lymphoproliferative disorders were the most frequent underlying disorder in $48 \%$ of cases, whereas myeloproliferative neoplasms and solid tumors accounted for $15 \%$ and $5 \%$ of cases. ${ }^{16-18}$ Mohri et al., in a prospective study evaluating 206 patients with a range of hematologic disorders, estimated that AvWS was present in approximately $10 \%$ of these patients. ${ }^{25}$ The different underlying disorders lead to AvWS through different mechanisms. In patients with hypothyroidism, the syndrome is caused by the decreased synthesis of an otherwise qualitatively normal vWF, and this can be reversed by 1 -thyroxine therapy. ${ }^{26,27}$ In most other cases, the synthesis of vWF in megakaryocytes and endothelial cells and its release in circulation are normal, so that AvWS recognizes other mechanistic pathways. In cardiac valvulopathies and left ventricular assist devices, sheering of high-molecular-weight (HMW) vWF multimers by mechanical stress or proteolysis induced by ADAMTS 13 (a disintegrin and metalloproteinase with a thrombospondin type 1 motif, member 13) are involved. ${ }^{28-33}$ In cases associated with plasma cell dyscrasias (MGUS and multiple myeloma), as well as in autoimmune diseases such as systemic lupus erythematosus, circulating autoantibodies directed against functional or non-functional vWF domains have been reported. ${ }^{31-33}$ Antibody binding to vWF leads to the formation of immune complexes that are cleared from the circulation by the reticulo-endothelial system. Antibodies that neutralize platelet-related vWF activities (inhibitors) have seldom been reported. Finally, a mechanism involving the selective adsorption of HMW multimers on tumor cells leading to their enhanced plasma clearance has been 
described in lymphoproliferative diseases (multiple myeloma, Waldenström's macroglobulinemia, nonHodgkin lymphoma, hairy cell leukemia) and solid cancers. ${ }^{33}$ In MGUS, the aberrant expression on abnormal plasma cells of the glycoprotein $\mathrm{Ib}$ (the principal platelet receptor of vWF) was associated with its selective binding to these cells. ${ }^{34} \mathrm{vWF}$ adsorption onto the cell membranes and subsequent plasma clearance has also been involved in AvWS associated with myeloproliferative neoplasms. ${ }^{35}$ For example, adsorption on platelets is the mechanism in essential thrombocythemia, with an inverse relationship between platelet count and the plasma defect of HMW multimers ${ }^{35}$ In addition, essential thrombocythemia and other myeloproliferative neoplasms may cause the syndrome through increased plasma vWF proteolysis.

\section{Clinical features}

The bleeding diathesis usually occurs rather late in life in persons with no past and family history of bleeding. The main symptoms are mild to moderately severe mucocutaneous bleeding (ecchymosis, epistaxis, menorrhagia, gastrointestinal tract bleeding), similar to inherited vWD, or excessive bleeding following trauma or surgical procedures, particularly when FVIII:C is low. Gastrointestinal bleeding is usually associated with the detection of angiodysplasia. The mechanism of this vascular abnormality, which has also been described in inherited type 2 and type $3 \mathrm{vWD}$, involves the defect of HMW vWF multimers, that characterizes all AvWS cases ${ }^{10}$ with the only exception of those associated with hypothyroidism. According to the ISTH registry, ${ }^{16-18}$ patients with AvWS associated with lymphoproliferative disorders have more severe bleeding symptoms than those with other underlying conditions, even though bleeding-related deaths have seldom been reported. As mentioned above, lymphoproliferative disorders account for a significant proportion of AvWS cases, ranging from $30 \%$ to $48 \%$. A few studies have investigated the association of myeloproliferative neoplasms with AvWS ${ }^{35-38}$ Mital et al., in two studies involving 312 consecutive patients with essential thrombocythemia $(n=170)$ or polycythemia vera $(n=142)$, found prevalences of $20 \%$ and $12 \%$, respectively, and they recommend that persons with these disorders and a bleeding tendency should be screened for the presence of an underlying AvWS. ${ }^{35,36}$ A high prevalence was also found by Rottenstreich et al. ${ }^{37}$ in 173 patients with thrombocythemia $(\mathrm{n}=116)$ or polycythemia $(\mathrm{n}=57),{ }^{37}$ meaning that $55 \%$ of those with thrombocythemia and $49 \%$ of those with polycythemia had an AvWS. Younger age, higher platelet count and hemoglobin levels, as well as the presence of the $J A K 2^{\mathrm{V} 617 \mathrm{~F}}$ somatic mutation, independently pre- dicted the syndrome in patients with thrombocythemia but not in polycythemia, the only predictor being a higher platelet count. ${ }^{37}$ Furthermore, Mital et al. ${ }^{38}$ in an investigation of 21 patients with systemic mastocytosis, found a high rate of AvWS accompanied by mild to moderate symptoms of mucocutaneous bleeding (ecchymosis, menorrhagia, epistaxis).

\section{Diagnosis}

In the absence of a family history of bleeding, the diagnosis of AvWS is usually based on the laboratory tests used to diagnose inherited $\mathrm{vWD} \cdot{ }^{39,40} \mathrm{~A}$ defect in primary hemostasis is demonstrated by a prolonged skin bleeding time or prolonged closure time with PFA-100.40 Plasma samples usually show normal or mildly decreased vWF:Ag contrasting with a more marked decrease in vWF:RCo and vWF:CB. ${ }^{40}$ The latter qualitative platelet-related activity assays of $\mathrm{vWF}$ are frequently lower than the quantitative vWF:Ag assay, resulting in vWF:RCo/vWF:Ag ratios that are often below 0.7 , similar to type $2 \mathrm{~A}$ vWD. ${ }^{40}$ When FVIII:C is low, a prolonged APTT is also observed. vWF multimer electrophoresis is warranted to demonstrate the defect of HMW multimers that helps to distinguish AvWS from type $1 \mathrm{vWD} .{ }^{9,10}$ Measurement of the plasma vWF propeptide, which reflects the degree of vWF biosynthesis, is not currently recommended to distinguish inherited vWD from AvWS ${ }^{41,42}$ because the latter is often characterized by an accelerated clearance of vWF from the circulation but normal synthesis, so that propeptide levels are normal. At variance with acquired hemophilia, autoantibodies that inhibit platelet-related VWF activity play a mechanistic role in a minority of AvWS cases. ${ }^{43,44}$ Nevertheless, because the presence of neutralizing antibodies seems to be associated with a more severe bleeding tendency, antibody screening should be performed in all AvWS cases. The most common method used to look for an inhibitory activity against vWF is based upon mixing studies (patient plasma mixed with normal plasma and incubated at $37^{\circ} \mathrm{C}$ ), followed by the measurement of both residual vWF:RCo and vWF:CB. ${ }^{40-44}$ However, an inhibitor is seldom demonstrated and the antibodies that bind vWF and accelerate its plasma clearance without neutralizing vWF activity cannot be detected with this method. Enzyme-linked immunosorbent assays (ELISA) are an option that has, however, not yet been adequately standardized. ${ }^{45}$ Overall, diagnosis of AvWS is difficult, and a correct workup should consider all information from both clinical and case history evaluation as well as laboratory results. In particular, the differential diagnosis with milder forms of inherited $\mathrm{vWD}$ is important given the difference in therapeutic approach (see below).

Table 1. Conditions associated with the acquired von Willebrand syndrome.

\section{Conditions}

Cancers

Hematologic: MGUS, multiple myeloma, Waldenstrom macroglobulinemia, chronic lymphocytic leukemia, hairy cell leukemia, lymphomas, essential thrombocythemia, polycythemia vera, chronic myeloid leukemia. Solid: Wilm's tumor, lung and bladder adenocarcinoma.

\begin{tabular}{ll} 
Autoimmune diseases & Systemic lupus erythematosus, autoimmune thyroid disorders, connective tissue diseases, GvHD. \\
\hline Drug-induced & Antibiotics (griseofulvin, ciprofloxacin), anticonvulsants (valproic acid), plasma volume expander (HES). \\
Other & $\begin{array}{l}\text { Cardiovascular disorders (aortic stenosis, congenital cardiac defects, mitral valve prolapse, left ventricular assist } \\
\text { devices), infections (viral, parasitical), uremia, gastrointestinal angiodysplasia, diabetes. }\end{array}$
\end{tabular}

MGUS: monoclonal gammopathy of unknown significance; GvHD: graft-versus-host disease; HES: hydroxyethyl starch. 


\section{Treatment}

There are three main treatment goals for patients with AvWS: the control of acute bleeding, its prevention in high-risk situations, and the achievement of a stable

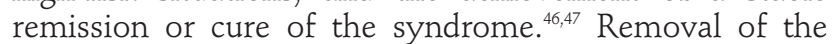
underlying disorder (i.e. by means of surgery, chemotherapy, radiotherapy and/or immunosuppressants), which is not within the scope of this review, is the only potentially curative approach. However, treatment of the associated condition may not be feasible, and a partial remission does not always ameliorate the bleeding symptoms.

As far as the hemostatic therapies are concerned, a range of different medications have been used: desmopressin (DDAVP), vWF/FVIII concentrates, antifibrinolytic agents, high-dose intravenous immunoglobulins (HDIVIg), plasmapheresis and recombinant activated factor VII (rFVIIa). ${ }^{46,47}$

The synthetic analog of DDAVP, given intravenously or subcutaneously at the dose of $0.3 \mu \mathrm{g} / \mathrm{kg}$, was used to control or prevent bleeding in the AvWS. ${ }^{48,49}$ The ISTH registry reported an overall success rate with DDAVP of approximately $30 \%$, although this varied according to the underlying disorder: lower in cardiovascular disorders $(10 \%)$ and myeloproliferative neoplasms $(21 \%)$, and higher in autoimmune $(33 \%)$ and lymphoproliferative $(44 \%)$ disorders. ${ }^{16-18}$ In a prospective trial performed in ten patients with MGUS, plasma vWF levels increased in all patients after DDAVP, but the increase was short-lasting. ${ }^{44} \mathrm{~A}$ short plasma half-life of vWF following DDAVP administration has also been observed in the context of other lymphoproliferative disorders and is thought to be related to the presence of anti-vWF antibodies. A transient effect of DDAVP was also observed in patients with myeloproliferative neoplasms, mainly due to adsorption or proteolytic cleavage of the released vWF. It is advisable to closely monitor FVIII:C and vWF:RCo/vWF:CB plasma levels after DDAVP in order to maintain these at levels that are sufficient to prevent or treat bleeds.

As far as replacement therapy is concerned, several plasma-derived concentrates containing vWF have been used in AvWS ${ }^{16-18}$ In the ISTH registry, vWF/FVIII concentrates showed a favorable response in approximately $40 \%$ of cases ${ }^{18}$ with dosages ranging between 30-100 vWF:RCo units $/ \mathrm{kg}$. The half-life of infused vWF is shorter than in inherited $\mathrm{vWD}$, thus requiring higher doses of vWF/FVIII concentrate to ensure adequate hemostatic plasma levels. ${ }^{16-18}$ Plasma FVIII:C and vWF:RCo must be monitored when administering DDAVP, particularly in patients undergoing invasive or surgical procedures.

The antifibrinolytic agents most commonly used in
AvWS are the lysine analogs $\varepsilon$-aminocaproic acid, administered at a dose of $50-60 \mathrm{mg} / \mathrm{kg}$ every $4-6$ hours, and tranexamic acid, at a dose of $20-25 \mathrm{mg} / \mathrm{kg}$ every $8-12$ hours. ${ }^{50}$ These drugs can be given orally, intravenously or topically, and act by inhibiting plasminogen activation. Antifibrinolytics are primarily used as adjuvants together with DDAVP or vWF/FVIII concentrates for surgery and bleeding, particularly at sites with high fibrinolytic activity (i.e. gastrointestinal and oral tracts); however, these drugs may be used alone for minor bleeding episodes.

The ISTH registry reported a 33\% success rate in clinical cases receiving HDIVIg in AvWS, especially for lymphoproliferative disorders $(37 \%)$, solid tumors $(100 \%)$, and immunological diseases $(50 \%){ }^{16-18}$ HDIVIg is especially useful in cases associated with MGUS. ${ }^{44}$ In a prospective trial which enrolled ten patients with AvWS and MGUS, HDIVIg was more effective than DDAVP and VWF/FVIII concentrates because there was a more sustained increase in FVIII/VWF activities and shortening of bleeding time for at least 15-20 days in all IgG-MGUS cases. ${ }^{44}$ In contrast, no response was observed in patients with IgM class MGUS (IgM-MGUS). ${ }^{44}$ In addition, prophylactic infusions of HDIVIg every 21 days stopped recurrent gastrointestinal bleeding in patients with IgG-MGUS. The mechanisms of action of HDIVIg may involve an anti-idiotype effect, blockage of reticulo-endothelial Fc-receptors, or capture of the circulating immune complexes by the immunoglobulins. ${ }^{53}$ The recommended doses of HDIVIg are $1 \mathrm{~g} / \mathrm{kg}$ for two days or $0.4 \mathrm{~g} / \mathrm{kg}$ for five days. ${ }^{51.54} \mathrm{The}$ increase in vWF and FVIII:C levels usually occurs within 24-48 hours and may last up to 3-4 weeks; thus, additional courses of HDIVIg every 21 days are necessary to maintain a clinical response. On the whole, HDIVIg represent an important therapeutic tool for the management of AvWS, especially for cases associated with $\operatorname{IgG}$ monoclonal gammopathies, lymphoproliferative disorders, and multiple myeloma associated IgG paraproteins. ${ }^{44}$ Due to the delay in the postinfusion increase in vWF and FVIII:C levels, HDIVIg is not effective in the management of acute bleeding; in these cases, the medication must be administered together with shorter-acting agents such as DDAVP and vWF/FVIII concentrates ${ }^{51.54}$ The main goal of plasmapheresis is to temporarily eliminate autoantibodies and paraproteins from the circulation. This procedure is particularly effective in IgM-MGUS, which generally responds poorly to other treatments. Finally, rFVIIa, usually administered at a dose of $90 \mu \mathrm{g} / \mathrm{kg}$ for a median of three doses, has been successfully used when bleeding associated with AvWS is unresponsive to DDAVP and vWF/FVIII concentrates. ${ }^{55.59}$

Table 2 summarizes the multiple therapeutic strategies available for the treatment or prevention of bleeding in

Table 2. Hemostatic therapies in acquired von Willebrand syndrome associated with different underlying diseases.

\begin{tabular}{lc} 
Underlying diseases & Therapy \\
Cardiovascular & vWF/FVIII concentrates, antifibrinolytics \\
Lymphoproliferative & HDIVIg \\
- IgG MGUS & Plasmapheresis, DDAVP, vWF/FVIII concentrates, antifibrinolytics, rFVIIa \\
- IgM MGUS & DDAVP, vWF/FVIII concentrates, antifibrinolytics, rFVIIa, HDIVIg \\
- Lymphoma, myeloma & DDAVP, vWF/FVIII concentrates, antifibrinolytics \\
\hline Myeloproliferative & HDIVIg, DDAVP, vWF/FVIII concentrates \\
Autoimmune &
\end{tabular}

MGUS: monoclonal gammopathy of unknown significance; vWF: von Willebrand factor; FVIII: factor VIII; HDIVIg: high-dose intravenous immunoglobulin; DDAVP: desmopressin; rFVIIa: recombinant activated factor VII. 
AvWS. DDAVP and vWF/FVIII concentrates typically give a prompt but short-lived improvement in vWF and FVIII levels. High doses of vWF/FVIII concentrates usually result in higher response rates, particularly in cases associated with lymphoproliferative disorders, which represent the most challenging condition to treat, and in the context of myeloproliferative neoplasms. In these cases, the short plasma residence time of vWF must be taken into account when dosages and intervals of administration of DDAVP and vWF/FVIII concentrates are planned. Patients with IgG autoantibodies or paraproteins usually respond to HDIVIg with longer-lasting effects. In addition, antifibrinolytics may be helpful as adjuvant therapy, particularly for the management of mucocutaneous bleeds.

\section{Conclusions}

Acquired von Willebrand syndrome is a highly heterogeneous bleeding disorder, usually characterized by mild to moderate hemorrhagic symptoms that may sometimes be severe, especially when the disease becomes manifest following surgery. Although AvWS is rare, our perception is that its true incidence is underestimated due to the diagnostic complexity. When laboratory findings suggest $\mathrm{vWD}$ in a patient with a negative personal and family history of a bleeding diathesis, possible AvWS-associated conditions should be explored. We advise screening for AvWS in those patients with conditions potentially associated with this syndrome, particularly onco-hematologic disorders, at the time of the onset of abnormal and otherwise unexplained bleeding and before undergoing invasive or surgical procedures.

The diagnostic workup of patients with suspected AvWS is challenging. This is mainly due to the overlap in clinical and laboratory features with those of inherited vWD. Diagnosis requires the close integration of hematologists with laboratory experts. Similarly, a close interaction between clinicians from different specialties (i.e. hematology, oncology, rheumatology, cardiology) is needed to appropriately manage patients with AvWS. Besides the control or prevention of bleeding, which often requires the combination of multiple hemostatic medications, the mainstay of treatment is the removal of the underlying conditions. When feasible, this is the only way to cure AvWS.

\section{References}

1. Michiels JJ, Michiels JJ, Budde U, et al. Acquired von Willebrand syndromes: clinical features, aetiology, pathophysiology, classification and management. Best Pract Res Clin Haematol. 2001;14(2):401-436.

2. Kumar S, Pruthi RK, Nichols WL. Acquired von Willebrand's syndrome: a single institution experience. Am J Hematol. 2003;72(4): 243-247.

3. Franchini M, Lippi G. Recent acquisitions in acquired and congenital von Willebrand disorders. Clin Chim Acta. 2007;377(1-2):6269.

4. Franchini M, Lippi G. Acquired von Willebrand syndrome: an update. Am J Hematol. 2007;82(5):368-375

5. Franchini M, Lippi G, Favaloro EJ. Advances in hematology. Etiology and diagnosis of acquired von Willebrand syndrome. Clin Adv Hematol Oncol. 2010;8(1):20-24

6. Mital A. Acquired von Willebrand syndrome. Adv Clin Exp Med. 2016;25(6):13371344.

7. Charlebois I, Rivard GE, St-Louis J. Management of acquired von Willebrand syndrome. Transfus Apher Sci. 2018;57(6): 721-723.

8. Menegatti M, Biguzzi E, Peyvandi F. Management of rare acquired bleeding disorders. Hematology Am Soc Hematol Educ Program. 2019;2019(1):80-86.

9. Leebeek FW, Eikenboom JC. Von Willebrand's disease. N Engl J Med. 2016;375(21):2067-2080

10. Mannucci PM. New therapies for von Willebrand disease. Blood Adv. 2019;3(21): 3481-3487.

11. Vincentelli A, Susen S, Le Tourneau T, et al. Acquired von Willebrand syndrome in aortic stenosis. N Engl J Med. 2003;349(4):343-349.

12. Loscalzo J. From clinical observation to mechanism--Heyde's syndrome. N Engl J Med. 2012;367(20):1954-1956.
13. Otto CM, Prendergast B. Aortic-valve stenosis--from patients at risk to severe valve obstruction. N Engl J Med. 2014;371(8):744 756.

14. Mehta R, Athar M, Girgis S, Hassan A, Becker RC. Acquired Von Willebrand Syndrome (AVWS) in cardiovascular disease: a state of the art review for clinicians. J Thromb Thrombolysis. 2019;48(1):14-26.

15. Horiuchi H, Doman T, Kokame K, Saiki Y, Matsumoto M. Acquired von Willebrand syndrome associated with cardiovascular diseases. J Atheroscler Thromb. 2019;26(4):303-314

16. Federici $\mathrm{AB}$, Rand $\mathrm{JH}$, Bucciarelli $\mathrm{P}$, et al. Acquired von Willebrand syndrome: data from an International registry. Thromb Haemost. 2000;84(2):345-349.

17. Federici $A B$, Budde $U$, Rand $J H$. Acquired von Willebrand syndrome 2004: international registry. Hämostaseologie. 2004;24(1):50 55 .

18. Federici $A B$, Budde U, Castaman G, Rand $\mathrm{JH}$, Tiede A. Current diagnostic and therapeutic approaches to patients with acquired von Willebrand syndrome: a 2013 update. Semin Thromb Hemost. 2013;39(2):191 201.

19. Muslem R, Caliskan K, Leebeek FWG. Acquired coagulopathy in patients With left ventricular assist devices. J Thromb Haemost. 2018;16(3):429-440

20. Thomas J, Kostousov V, Teruya J. Bleeding and thrombotic complications in the use of extracorporeal membrane oxygenation. Semin Thromb Hemost. 2018;44(1):20-29.

21. Coppola A, Favaloro EJ, Tufano A, Di Minno MND, Cerbone AM, Franchini M. Acquired inhibitors of coagulation factors: Part Iacquired hemophilia A. Semin Thromb Hemost. 2012;38(5):433-446.

22. Franchini M, Mannucci PM. Acquired haemophilia: a 2013 update. Thromb Haemost. 2013;110(6):1114-1120.

23. Tiede A, Abdul Karim F, Jiménez-Yuste V, et al. Factor VIII activity and bleeding risk dur- ing prophylaxis for severe hemophilia A: a population pharmacokinetic model. Haematologica. 2020 Apr 23. doi 10.3324/haematol.2019.241554. [Epub ahead of print]

24. Veyradier A, Jenkins CS, Fressinaud E, Meyer D. Acquired von Willebrand syndrome: from pathophysiology to management. Thromb Haemost. 2000;84(2):175-182.

25. Mohri H, Tanabe I, Ohtsuka M, et al. Acquired von Willebrand disease associated with multiple myeloma: characterization of an inhibitor to von Willebrand factor. Blood Coagul Fibrinolysis. 1995;6(6):561-566.

26. Bruggers CS, McElligott K, Rallison ML. Acquired von Willebrand disease in twins with autoimmune hypothyroidism: Response to desmopressin and L-thyroxine therapy. J Pediatr. 1994;125(6 Pt 1):911-913.

27. Franchini M, de Gironcoli M, Lippi G, et al Efficacy of desmopressin as surgical prophylaxis in patients with acquired von Willebrand disease undergoing thyroid surgery. Haemophilia. 2002;8(2):142-144.

28. Tsai HM, Sussman II, Nagel RL. Shear stress enhances the proteolysis of von Willebrand factor in normal plasma. Blood. 1994;83(8): 2171-2179.

29. Yoshida K, Tobe S, Kawata M, Yamaguchi M. Acquired and reversible von Willebrand disease with high shear stress aortic valve stenosis. Ann Thorac Surg. 2006;81(2):490-494.

30. Pareti FI, Lattuada A, Bressi C, et al Proteolysis of von Willebrand factor and shear stress-induced platelet aggregation in patients with aortic valve stenosis Circulation. 2000;102(11):1290-1295.

31. Mohri H, Motomura S, Kanamori $\mathrm{H}$, et al Clinical significance of inhibitors in acquired von Willebrand syndrome. Blood. 1998;91 (10):3623-3629

32. Gan TE, Sawers RJ, Koutts J. Pathogenesis of antibody-induced acquired von Willebrand syndrome. Am J Hematol. 1980;9(4):363 371

33. Richard C, Cuadrado MA, Prieto M, et al. 
Acquired von Willebrand disease in multiple myeloma secondary to absorption of von Willebrand factor by plasma cells. Am J Hematol. 1990;35(2):114-117.

34. Scrobohaci ML, Daniel MT, Levy Y, Marolleau JP, Brouet JC. Expression of GpIb on plasma cells in a patient with monoclonal IgG and acquired von Willebrand disease. $\mathrm{Br}$ I Haematol. 1993;84(3):471-475.

35. Mital A, Prejzner W, Bieniaszewska M, Hellmann A. Prevalence of acquired von Willebrand syndrome during essential thrombocythemia: a retrospective analysis of 170 consecutive patients. Pol Arch Med Wewn. 2015;125(12):914-920.

36. Mital A, Prejzner W, ŚwiątkowskaStodulska R, Hellmann A. Factors predisposing to acquired von Willebrand syndrome during the course of polycythemia vera - retrospective analysis of 142 consecutive cases. Thromb Res. 2015:136(4):754-757.

37. Rottenstreich A, Kleinstern G, Krichevsky S, Varon D, Lavie D, Kalish Y. Factors related to the development of acquired von Willebrand syndrome in patients with essential thrombocythemia and polycythemia vera. Eur J Intern Med. 2017:41:49-54.

38. Mital A, Prejzner W, Hellmann A. Acquired von Willebrand syndrome during systemic mastocytosis: an analysis of 21 cases. Pol Arch Intern Med. 2018;128(7):491-493.

39. Federici AB. Acquired von Willebrand syndrome: is it an extremely rare disorder or do we see only the tip of the iceberg? J Thromb Haemost. 2008;6(4):565-568.

40. Favaloro EJ, Facey D, Grispo L. Laboratory assessment of von Willebrand factor. Use of different assays can influence the diagnosis of von Willebrand's disease, dependent on differing sensitivity of sample preparation and differential recognition of high molecular weight VWF forms. Am I Clin Pathol. 1995;104(3):264-271.

41. van Genderen PJ, Boertjes RC, van Mourik
JA. Quantitative analysis of von Willebrand factor and its propeptide in plasma in acquired von Willebrand syndrome. Thromb Haemost. 1998;80(3):495-498.

42. Eikenboom J, Federici AB, Dirven RJ, et al; MCMDM-1VWD Study Group. VWF propeptide and ratios between VWF, VWF propeptide, and FVIII in the characterization of type 1 von Willebrand disease. Blood. 2013;121(12):2336-2339.

43. Fricke WA, Brinkhous KM, Garris JB, Roberts HR. Comparison of inhibitory and binding characteristics of an antibody causing acquired von Willebrand syndrome: an assay for von Willebrand factor binding by antibody. Blood. 1985;66(3):562-569.

44. Mannucci PM, Lombardi R, Bader R, et al. Studies of the pathophysiology of acquired von Willebrand's disease in seven patients with lymphoproliferative disorders or benign monoclonal gammopathies. Blood. 1984;64(3):614-621

45. Siaka C, Rugeri L, Caron C, Goudemand J. A new ELISA assay for diagnosis of acquired von Willebrand syndrome. Haemophilia. 2003;9(3):303-308.

46. Federici AB. Therapeutic approaches to acquired von Willebrand syndrome. Expert Opin Investig Drugs. 2000;9(2):347-354.

47. Tiede A, Rand JH, Budde U, Ganser A, Federici $\mathrm{AB}$. How I treat the acquired von Willebrand syndrome. Blood. 2011;117(25): 6777-6785.

48. Franchini M. The use of desmopressin as a hemostatic agent: a concise review. Am J Hematol. 2007:82(8):731-735.

49. Biguzzi E, Siboni SM, Peyvandi F. Acquired von Willebrand syndrome and response to desmopressin. Haemophilia. 2018;24(1):e25e28

50. Franchini M, Mannucci PM. The never ending success story of tranexamic acid in acquired bleeding. Haematologica. 2020;105 (5):1201-1205.
51. Federici AB. Use of intravenous immunoglobulin in patients with acquired von Willebrand syndrome. Hum Immunol. 2005;66(4):422-430

52. Federici AB, Rossi V, Sacchi E, Franchini M Are intravenous immunoglobulins really inappropriate in acquired von Willebrand syndrome? Blood Transfus. 2012;10(3):402403.

53. Stone ME, Mazzeffi M, Derham J, Korshin A. Current management of von Willebrand disease and von Willebrand syndrome. Curr Opin Anaesthesiol. 2014;27(3):353-358.

54. Michiels JJ, van Vliet HH. Acquired von Willebrand disease in monoclonal gammapathies: Effectiveness of high-dose intravenous gamma globulin. Clin Appl Thromb Hemost. 1999;5(3):152-157.

55. Colella MP, Duarte GC, Marques JF Jr, De Paula EV. Haemostatic management of extreme challenges to haemostasis in acquired von Willebrand syndrome. Haemophilia. 2012;18(3):e188-191.

56. Friederich PW, Wever PC, Briët E, Doorenbos CJ, Levi M. Successful treatment with recombinant factor VIIa of therapyresistant severe bleeding in a patient with acquired von Willebrand disease. Am J Hematol. 2001;66(4):292-294.

57. Franchini M, Veneri D, Lippi G. The use of recombinant activated factor VII in congenital and acquired von Willebrand disease. Blood Coagul Fibrinolysis. 2006;17(8):615619.

58. Smaradottir A, Bona R. A case of acquired von Willebrand syndrome successfully treated with recombinant factor VIIa during thyroidectomy. Thromb Haemost. 2004;92(3): 666-667.

59. Karger R, Weippert-Kretschmer M, Budde $\mathrm{U}$, Kretschmer V. Diagnosis and therapeutic management in a patient with type 2B-like acquired von Willebrand syndrome. Blood Coagul Fibrinolysis. 2011;22(2):144-147. 